\title{
Innovation in Architecture Education: Collaborative Learning Method Through Virtual Reality
}

\author{
Hozan Latif Rauf \\ Department of Architectural Engineering, Tishk International University-Erbil, \\ Kurdistan Region, Iraq \\ Sardar S. Shareef \\ Department of Architectural Engineering, Tishk International University-Erbil, \\ Kurdistan Region, Iraq \\ Nizar Najim Othman \\ Department of Architectural Engineering, Tishk International University-Erbil, \\ Kurdistan Region, Iraq
}

\begin{abstract}
Architecture education is recognised for its collaborative approach, which stems from apprenticeships and design studio contexts. Furthermore, architecture education is a visually oriented profession that combines imagination. Virtual reality is now one of the techniques utilised in architectural education in various ways in this technology epoch; dealing with virtual reality devices or operating in virtual reality settings are both options. This article is based on a literature review of virtual reality encounters and long-term collaborative learning approaches. Thus, it is analytic and literature-based research. The goal is to look at how virtual reality might help with cooperation in architectural classes.
\end{abstract}

Keywords: architecture education, virtual reality, collaboration

\section{INTRODUCTION}

Prior to establishing the Ecole des Beaux-Arts in France, master apprenticeship was the sole way of training architects. However, this comprehensive method, which included painting, sculpture, and carving in addition to architectural teaching, could not survive the demands of the French government for long, and as a consequence, it became autonomous in 1863 and reformed higher education. Following that era, the Ecole des Beaux-Arts underwent a major transformation, bringing together architectural students and integrating them into an academically-focused establishment in the manner of a school.

The second pathway began with the foundation of Bauhaus in Germany (Gülay Taşç1, 2016). The two schools' methodologies have influenced architectural education in various ways. Bauhaus is regarded as the most radical revolution in architectural education, as evidenced by integrating art and craft into the curriculum and various other techniques spread out across the school's study time. Both institutions emphasised the construction-based technique and problem-oriented learning research (Gülay Taşçı, 2016). 
Furthermore, since the period of traditional schools, the project-based technique has been the primary path. The approach necessitates group work, hands-on experience with projects while modelling and sketching in studios, and face-to-face contacts.

Throughout all of the changes brought about by technological advancements, design cooperation remained essential and unavoidable. Furthermore, as an innovative method, technical developments provided additional paths from actual collaboration to virtual collaboration through digital technology. As a result, architects and other related parties are now practising and interacting differently (Gül, Wang, Bülbül, Çağdaş, \& Tong, 2008).

This paper aims to unfold the possibilities of virtual reality in activating all described collaboration levels and describe the potential of virtual reality as a part of nowadays teaching and learning tools in architecture education.

\section{Collaboration Levels in Architecture}

Collaboration can be found on many levels in architecture education studies. As are explained below:

1. Student-instructor collaboration: this kind of collaboration is a common way of transferring knowledge from instructors. A friendly environment might enhance and facilitate the learning process. Also, applying the active learning methods either in classes and studios will assist the process to be more effective, making students more active learners. The collaboration process depends on how the instructor presents him/herself concerning social subjects inside the class/studio and outside.

2. Student-student collaboration: student collaboration is another considerable way students learn from each other during their presence in classes or studios. Especially in a highly social environment, students learn how to interact, critique, engage in debate, and work together (Lueth, 2008). Students spend most of their time together; that is why it is expected to influence each other. Transferring knowledge occurs in different levels of interaction.

3. Student-client collaboration: students mostly pick imaginary client profiles during their projects' designs because they may not have the chance to learn how to interact and collaborate with real clients. If students can design for real clients, they will establish their future communication foundation as an architect as design cannot be an architects' only concept outcome regardless of the clients' opinion. This kind of architect-client interaction can be formulated in the form of communication and for having a healthy communication between them, as Shen (2011) proposed these points:

- The client should contribute to the design process and give him/her the sense that the contribution is valued.

- The client has the right to administer the design changes according to the requirements.

- Providing design visualisation techniques for the sake of a proper understandable process.

4. Student-industry collaboration: industry can be one of the other fields in which architectural students can collaborate via visiting factories of the building component manufacturers, learning from the visual-based experience and collaborating with the technical staff. From that, students will construct their knowledge through a social constructivist approach to learning.

\section{Virtual Reality}

Mandal (2013) defines virtual reality as a computer-simulated setting, whether a simulation of the actual world or an imaginative one, that enables users to interact. It is essential to understand, feel, and connect the past, present, and future. Moreover, Pope (2018) describes VR as:

The employment of technology to generate the illusion of existence in a non-existent environment is known as virtual reality. It operates by tricking our brains into believing we perceive something virtual by delivering information to multiple senses, including sight and sound. The illusion is frequently enhanced by the existence of interactivity, which means that the virtual environment reacts to your existence in some manner. 
Based on the previous definitions, virtual reality may be defined as a new kind of computer technology that allows humans to sense other worlds and experience things that do not prevail physically in VR version displays. However, there are some changes to the theme that make humans feel more in touch with reality.

Virtual reality as a notion is not unfamiliar; it is said to have originated in the early nineteenth century with panoramic 360-degree paintings. The human mind may be captivated and feel as if it is there in historical events in these artworks. Charles Wheatstone studied these two-dimensional pictures in 1838 and discovered a way to let the human mind view single images in three dimensions. He spoke on the topic of stereoscopic photography. View-Master stereoscopic pictures were utilised as "virtual tourism" after that, starting in 1939. Molten Heilig's Sensorama was the first machine designed in the 1950s that included simulations of all human sensations and produced a completely immersed experience for those who watched the machine's specialised films (URL-1, 2021).

FIGURE 1

SENSORAMA MACHINE

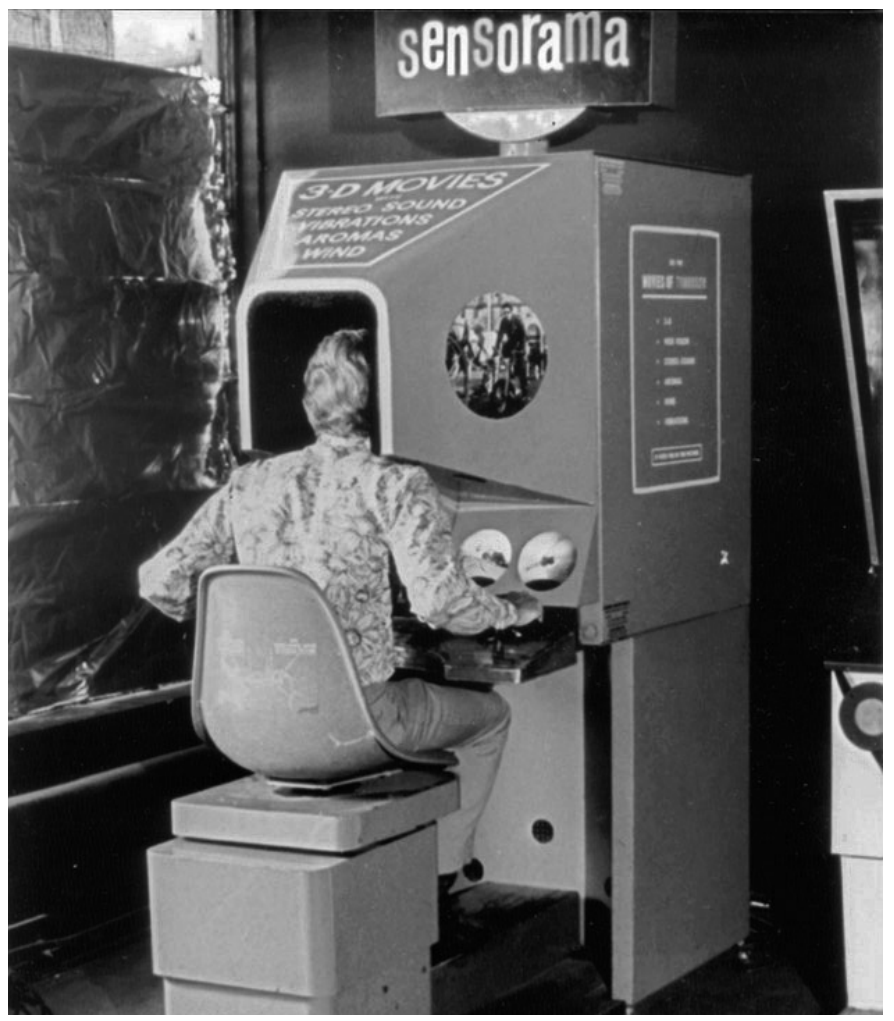

SOURCE: (URL-1, 2021)

The 'ultimate display' was one of the most well-known studies on the subject. It was first used in 1965 by Ivan Sutherland in a seminal work to show the sensory input and output that are the foundations of today's virtual science (Gobbetti \& Scateni, 1998).

The notion of virtual reality then achieved its pinnacle in the twentieth and twenty-first centuries, particularly in the video gaming industries, as new technology advanced at a breakneck pace. Virtual reality, in some cases, became a simple product in hand, including DIY headsets and Google's cardboards that are employed with Samsung smartphones, and has become widespread in many educational institutions as a result of all these devices and new technologies.

\section{Virtual Reality Types}

According to Wong (1996), virtual reality systems are divided into three categories: 
1. Things are shown in $2 \mathrm{D}$ on the monitor via sophisticated stored data in the computer, giving virtually genuine visuals, sound, and responsiveness. Humans can engage with the system and are considered to be genuine. This is primarily used in computer games.

2. Immersive systems: These systems use a Head-Mounted Display (HMD) in the form of a helmet or a customised mask to completely immerse humans in the virtual world. It may be observed when individuals wish to perceive an area and move through it virtually.

3. Telepresence: Unlike the former two, this technology combines real-world remote sensors with a human driver's senses (Wong, 1996).

Some organisations use this method to programme robots with remote human senses. For example, NASA plans to deploy telerobots to Mars, providing the impression that humans are virtually on Mars (Goldberg, 2001).

Meanwhile, Mazuryk and Gervautz (1996) introduce a new form of virtual reality, cyberspace, initially utilised by William Gipson. A collective illusion shared by billions of genuine operators daily like a visual depiction of data derived from each computer in the human system.

\section{Virtual Reality in Education}

Virtual reality has become an important topic in several very sensitive areas. Mantovani (2001) claims that the goal is to develop and use virtual reality to enhance the learning experience, not determine if VR is beneficial for education. Because for several years, augmented reality has been utilised in the army, law enforcement, and research facilities for training and teaching (Stevens \& Eifert, 2014). Virtual reality is the practise environment they must begin in, just as it is in pilot training and military training.

Furthermore, there are efforts to include VR at the beginning of education, particularly in schools, to offer pupils stronger and clearer imaginations. Fineman (2017) categorises the real-world uses of virtual reality in education as follows:

- Field Trips: Students get the opportunity to see locations that are difficult to travel in people, such as the Amazon Forest or the surface of Mars.

- Training: Students may practise and experience the subjects they study, such as archaeological dig sites, aviation landings, or surgical operations, thanks to VR technology.

- Recruiting: Some institutions utilise this approach to provide virtual campus visits to students. Other institutions use it to lure players by giving them the same experience as playing in front of thousands of spectators in a real huge auditorium.

- Design: It is one of the most practical alternatives for architectural education. It can show students' buildings before they are built, allowing them to explore information.

- Distance Learning: Faculty and students at Penn State have stated that conventional online techniques enhanced by virtual reality enhance learning outcomes. The Stanford School of Business also offers credentials based on virtual reality.

- Collaboration: Collaboration via VR has much potential now since it reduces the sensation of distance because humans can interact without having to be in the same area, thus not just for research and teaching. An Immersive Virtual Environment at University College London illustrates how cooperative virtual avatars with physical environments may apply augmented reality. Another example is Case Western Reserve University, which has collaborated with Microsoft to use augmented reality to convey medical information to the Hololens.

\section{Techniques for Incorporating Virtual Reality Into Architecture}

One of the disciplines that could benefit from virtual reality is architecture. The earliest use of virtual reality technology was to assist architecture students with visualising in three dimensions, which is probably the most challenging aspect of learning architecture (Sala, 2006). This is one method of impacting VR on architectural education through 3D software applications, which has influenced the architecture education curriculum by including these programmes in the design.

Virtual reality also contributes to the creation of virtual worlds through the use of headgear and computer simulations. It offers several advantages, such as allowing pupils to see every design aspect in a 
separate environment known as the virtual world. In the virtual world, as Mateu, Lasala, and Alamán (2015) describe, knowledge is gained through 'learning by doing' in a safe atmosphere with a sense of immersion in a realistic situation (Mateu et al., 2015). Students engage in a Vygotskian learning environment wherein they gain information in a semi-sensual physical world and interact with $3 \mathrm{D}$ objects.

In their study, Hussein and Nätterdal (2015) stated that the objective of virtual reality is to enrich, encourage, and excite students' understanding of certain events while also allowing them to engage in hands-on learning.

Even using virtual reality in education addresses many problems that are tough to tackle in the actual world. Thus, Virtual-Reality is more attractive because it can be used to replicate and allow students to practise ways without jeopardising their safety, and therefore may be utilised in traditional educational situations where teaching students is difficult (Hussein \& Nätterdal, 2015).

Several studies have shown that students are enthusiastic about learning in virtual reality and that virtual reality in education may create a change by introducing students to new insights, inspiring, motivating, and thrilling them. They should be allowed to engage in the educational environment, allowing them to feel a feeling of belonging (Pantelidis, 2010).

For this reason, Pantelidis (2010) cites the essential justifications for utilising virtual reality in education:

- Virtual reality takes advantage of the potential of visual representations to develop new forms and methods of visualisation. As a result, it provides a unique method of displaying information. In some cases, virtual reality (VR) can portray certain characteristics, processes, and occurrences more realistically than other approaches, allowing for remarkable close-up inspection of an object, tracking from a great distance, and analysing and evaluating places and events not available by other techniques.

- Students are motivated by virtual reality. It fosters active rather than passive involvement and necessitates contact. Some virtual reality systems, such as collaborative virtual reality, combine text input with virtual environments, encouraging or requiring cooperation and fostering a social environment.

- Students can go through an adventure in virtual reality at their own pace over an extended period without being constrained by a traditional class schedule. It allows disabled persons to participate in research or learning activities when they would otherwise be unable to. It is unhindered by language barriers. Individuals may speak with pupils from other cultures on an equal footing and play with people from different cultures using VR with text accessibility (Pantelidis, 2010).

VR has a wide range of applications in the sphere of education thus far. Education in architecture may not be an exception.

\section{Virtual Reality and Collaboration in Architecture}

In architecture, virtual reality has become the most popular technique to improve a structure's perception that would not exist. Researchers are already experimenting with virtual reality as a medium for design creation and discovery. It has also been extensively utilised in the design to envision various design alternatives. It is significantly easier and less expensive to analyse a design in Virtual Reality (Abdelhameed, 2013).

In order to focus on architectural collaboration in the information age, it is crucial to look at the possibilities of virtual reality (VR) to see how it affects and improves the collaborative effort in architecture. The concept of 'virtual touch' is a cutting-edge issue in the VR industry today, and it is the most difficult subject for students and instructors to utilise. As previously said, including visual and audio elements has a long history, yet touch has stayed absent from VR simulations. People may now alter 3D graphics by touching them on touchable screens, thanks to new technologies. Virtual Touch is a mixed reality technology that allows students to build 3D collaborative spaces in a virtual world to participate in problemsolving actively. Students can organise workgroups in which they might create and share information. Furthermore, Virtual Touch's tangible interfaces enable the handling of physical objects that has 
consequences in the virtual world. With a learning situation, the student should investigate, experiment, and engage in the virtual world utilising physical manipulation, therefore seeing the direct repercussions of his/her activities (Mateus et al., 2015).

Functioning in a collaborative setting might add a game-like element to the learning process. Who are the pupils who are purposefully prepared to receive information without being stressed, due to the wide range of applications and uses of virtual reality in all disciplines of education, including architecture, this article summarises the issue of virtual reality's potential in design studios in terms of a collaborative learning paradigm, which is:

1. Virtual design studio: During its time in education, the design studio is well-known for its collaborative approach. Nevertheless, even though possessing a virtual design studio is believed to be one side of the coin, interactions in design studios will be altered during VR. The Virtual Design Studios (VDS) examines asynchronous and synchronous remote design collaboration techniques, according to (Reffat, 2005). Students acquire a better grasp of new modalities of cooperation and media incorporation in design practises by employing technologies including video conferencing, Internet publishing, email, Web3D, and digital modelling. This technique will be useful for collaborative learning amongst institutions in different regions. In 2007, the University of Sydney and the Istanbul Technical University put this approach to the test. Fifty-two students completed a joint-design project in a collaborative virtual environment as part of the curriculum. According to the findings, kids may be exposed to a new learning style and profit from it. Teleconferences, online chats, emails, and text messaging are all important components of the process (Gül et al., 2008).

2. Studio design in virtual environments: studios exist in actual form in this technique. In these studios, students may learn without using paper. This is accomplished by supplying headsets and lenses and virtual-based software to the new virtual environment makers. This allows anybody to contribute and go through the designs. Virtual learning has much promise with this technique of teaching and learning. Students may look at the results of their drawings and their faults and details (Anderson, Esser, \& Interrante, 2003). However, Schnabel (2004) reports that IVEs (Internet Virtual Environments), which allow users to interact with space and volume in three dimensions, will not be frequently employed in the design stage. Only a few imaginative illustrations use IVE in the design process, like movies, games, or big ventures like aeronautics. It is thought that, in today's world, the process has accelerated due to fast technological advancements. That is why virtual environment design has become a popular topic in architecture education in some parts of the world.

\section{DISCUSSION}

Virtual reality and technology have now become de facto in people's daily lives. All of the above claims, including architectural education, have interacted with the educational process. Virtual reality cannot be drawn out in the educational process in many sectors. While the profession has a tradition of genuine faceto-face, tactile, touchable activity, this has distinct connotations for architectural education. Designing in virtual worlds raises the question of whether the new VR idea influences the teaching and learning process in any manner.

In the collaboration mentioned above levels in architecture, virtual reality influences each level, for instance in:

- Student-instructor level: virtual reality can promote learning in this stage by engaging to online social media groups such as Facebook, Viber, and video conferences. Instructors can change the sense of place by flipping the meaning of existing in the classrooms called 'flipped classes' instead of giving lectures in classrooms, instructors can send the required stuff online, and the classroom becomes the place for discussing what would have been sent.

- Student-student: in the same method, students can collaborate in social media groups, transferring knowledge and keeping themselves up to date regarding the study materials and 
social relationships. By providing VR environments in the studio, they will have the chance to observe the projects via VR lenses and gadgets.

- Student-client: clients need more attention concerning the understanding of the design. That is why having strong VR media is necessary. So, the clients can keep their understanding and perception at a high level. In this way, regardless of the 3D software programs used by students, it may put clients into a virtual environment being a better way.

- Student-industry: many industries have a developed advertisement method which virtual reality might incorporate. In this way, students can observe and see the products' details, making them more knowledge-based and logical during design. Such as what IKEA provided, has 2D/3D and BIM catalogue and is accessible to all users in the designs in any view or direction they may prefer (Niedermair, 2012).

The above real-world applications of virtual reality in education raise questions: why do education organisations and students still require real-world face-to-face interaction in the teaching-learning activities in physical classrooms and studios if VR has quite an impactful participation in education? Is sustaining social connections and living together with a human need? Is it a human habit that cannot be broken or what?

\section{CONCLUSION}

Collaboration is one of the most important topics in architectural education, which dates back to the apprenticeship period and includes design studios. Deprivation and isolation are two negative beliefs about new technology, yet virtual reality developments are meant to maintain collaboration on certain levels. In addition, Constructivist (constructivism) and contextual learning theories promote the integration of VR into architectural instruction. Nonetheless, virtual reality has had such a beneficial impact on architectural education that it appears that architecture education will be impossible to proceed without face-to-face realworld design settings.

\section{ACKNOWLEDGEMENT}

We wish to show our appreciation to Tishk International University, Erbil, Iraq, as this research is supported by its Research Center.

\section{REFERENCES}

Abdelhameed, W.A. (2013). Virtual Reality Use in Architectural Design Studios: A case of studying structure and construction. Procedia Computer Science, 25, 220-230.

Anderson, L., Esser, J., \& Interrante, V. (2003). A virtual environment for conceptual design in architecture. Paper presented at the Proceedings of the Workshop on Virtual environments 2003.

Fineman, B. (2017). Real Applications of Virtual Reality in Education.

Gobbetti, E., \& Scateni, R. (1998). Virtual reality: Past, present, and future. Virtual environments in clinical psychology neuroscience: Methods techniques in advanced patient-therapist interaction.

Goldberg, K. (2001). The Robot in the Garden: Telerobotics and Telepistemology in the Age of the Internet. Mit Press.

Gül, L.F., Wang, X., Bülbül, T.T., Çağdaş, G., \& Tong, H. (2008). Global teamwork: A study of design learning in collaborative virtual environments.

Gülay Taşçı, B. (2016). Theoretical frame work for using digital games in architecture education. Architecture Research, 6(3), 51-56.

Hussein, M., \& Nätterdal, C. (2015). The benefits of virtual reality in education - A comparision Study.

Lueth, P.L.O. (2008). The architectural design studio as a learning environment: A qualitative exploration of architecture design student learning experiences in design studios from firstthrough fourth-year. Iowa State University. 
Mandal, S. (2013). Brief introduction of virtual reality \& its challenges. International Journal of Scientific Engineering Research, 4(4), 304-309.

Mantovani, F. (2001). 12 VR learning: Potential and challenges for the use of $3 d$ environments in education and training. Towards Cyberpsychology: Mind, Cognition, Society in the Internet Age, $2(207)$.

Mateu, J., Lasala, M.J., \& Alamán, X. (2015). Developing mixed reality educational applications: The virtual touch toolkit. Sensors, 15(9), 21760-21784.

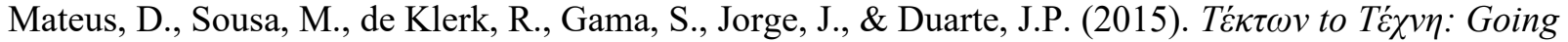
back to the Classical Roots of Architecture using Virtual Reality. Paper presented at the eCAADe.

Mazuryk, T., \& Gervautz, M. (1996). Virtual reality-history, applications, technology and future.

Niedermair, S. (2012). Augmented reality on mobile devices for architectural visualisation.

Pantelidis, V.S. (2010). Reasons to use virtual reality in education and training courses and a model to determine when to use virtual reality. Themes in Science Technology Education, 2(1-2), 59-70.

Pope, H. (2018). Introduction to virtual and augmented reality. Library Technology Reports, 54(6), 5-7.

Reffat, R.M. (2005). Collaborative digital architectural design learning within $3 D$ virtual environments.

Sala, N. (2006). Multimedia and virtual reality in architecture and in engineering education. Paper presented at the Proceedings of the 2nd WSEAS/IASME International Conference on Educational Technologies, Bucharest, Romania.

Schnabel, M.A. (2004). Architectural design in virtual environments: Exploring cognition and communication in immersive virtual environments.

Shen, W. (2011). A BIM-based Pre-occupancy Evaluation Platform (PEP) for facilitating designer-client communication in the early design stage.

Stevens, J., \& Eifert, L. (2014). Augmented reality technology in US army training (WIP). Paper presented at the Proceedings of the 2014 Summer Simulation Multiconference.

URL-1. (2021). History Of Virtual Reality. Retrieved from https://www.vrs.org.uk/virtualreality/history.html

Wong, G. (1996). The Philosophy of Virtual Reality. VR, 96. 